\title{
DESARROLLO PROFESIONAL DEL PROFESOR DE LENGUAS EXTRANJERAS A TRAVÉS DE LA COLABORACIÓN TRANSNACIONAL Y EL USO DE LAS TIC
}

\author{
Jaume Batlle Rodríguez \\ Azahara Cuesta García \\ $M^{a}$ Vicenta González Argüello \\ Universidad de Barcelona
}

\begin{abstract}
RESUMEN: Actualmente, en un mundo en constante evolución, una de las premisas en la formación de profesores es la necesidad de fomentar un perfil de docente en desarrollo profesional continuo. Los futuros profesores necesitan estrategias formativas para adquirir herramientas y habilidades que les capaciten para una formación autónoma a lo largo de la vida. Esta formación se lleva a cabo en una realidad social en la que las tecnologías tienen cada vez más un papel preponderante. Este artículo tiene como objetivo presentar un proyecto de innovación docente transnacional para el desarrollo profesional continuo de profesores de lenguas extranjeras a través de acciones formativas basadas en el desarrollo de la práctica reflexiva y el uso de las tecnologías móviles. Los primeros resultados muestran que el carácter transnacional del proyecto formativo y el foco pedagógico dirigido hacia la práctica reflexiva y el uso de las TIC en las estancias internacionales han permitido a los profesores en formación adquirir destrezas formativas, como un uso más eficiente de las tecnologías móviles o una mayor capacitación para enfocar la práctica reflexiva como herramienta para la formación continua.
\end{abstract}

PALABRAS CLAVE: Desarrollo Profesional Continuo, enseñanza de lenguas extranjeras, innovación docente, formación de profesores, tecnologías móviles.

\section{FOREIGN LANGUAGE TEACHER PROFESSIONAL DEVELOPMENT THROUGH TRANSNATIONAL COLLABORATION AND THE USE OF ICT}

\footnotetext{
ABSTRACT: At present, in a constantly evolving world, one of the underpinned premises in teacher training is the need for teachers to be aware that they should take on a Continuous Professional Development. Future teachers need training strategies to acquire tools and skills that allow them
} 
to follow a life-long learning process. This training is carried out in a social reality where new technologies are increasingly adopting a major role. This article has the aim to present a transnational training innovation project for foreign language teachers' Continuing Professional Development through teaching actions based on the development of reflective practice and the use of mobile technologies. First results show that this training project has enabled to pre-service teachers to acquire teaching skills, such as a more efficient use of mobile technologies or a better capacity to focus on reflective practice as a tool for Continuing Professional Development.

KEYWORDS: Continuing Professional Development, foreign language teaching, teaching innovation, teacher training, mobile technologies.

Recibido: 24/07/2019

Aceptado: 13/04/2020

Correspondencia: Jaume Batlle Rodríguez PhD. Facultat d'Educació, Universitat de Barcelona, Mundet, Edifici Llevant, despatx 132 Pg. Vall d'Hebron, 171. 08035 Barcelona. Email: jaumebatlle@ub.edu.

\section{INTRODUCCIÓN}

En la actualidad, los profesores saben que la finalización de los estudios de grado o máster no es la finalización de su etapa formativa. En una sociedad líquida y en constante cambio, la formación de profesores se ha convertido en una necesidad que va más allá de las fronteras espacio-temporales de la universidad. Entre otros aspectos, el Desarrollo Profesional Continuo (DPC, en adelante) requiere encaminar a los docentes en formación hacia procesos de reflexión acerca de la propia realidad profesional, el desarrollo de habilidades y competencias que les permitan ser flexibles y adaptables a las nuevas realidades académicas y el dominio competencial de las Tecnologías de la Información y la Comunicación (TIC). Estos procesos reflexivos les capacitarán para valorar de forma fundamentada su usabilidad para el aprendizaje. Fomentar el DPC permite que los profesores estén actualizados profesionalmente en todo momento en la medida de lo posible. No obstante, su implementación debe venir acompañada de una oferta formativa adecuada para ello. En un mundo cada vez más global, más conectado y más mediado por la tecnología, una formación transnacional se antoja pertinente para la formación de profesores de siglo XXI y para un desarrollo profesional centrado en la práctica reflexiva, una orientación investigativa y el uso activo, creativo y reflexivo de las TIC.

El presente artículo tiene como objetivo presentar el proyecto PROPIC Europa', un proyecto para docentes en formación inicial desarrollado con la intención de

1. PROPIC Europa: Promoting Professionalism, Innovation and Transnational Collaboration in Teacher Education. Proyecto Erasmus + KA2. Código: 2017-1-DE01-KA203-003547 IP: Dr. Götz Schwab (Paedagogishe Hochschule Karlsruhe, Alemania). 
promover el DPC a través de una experiencia práctica fundamentada en la colaboración transnacional, la investigación, la práctica reflexiva y el uso de las TIC. El proyecto ha sido implementado por parte de cinco instituciones europeas de educación superior en el ámbito de la formación de profesores de lenguas extranjeras: Paedagogishe Hochschule Karlsruhe (Alemania), Christian Albrechts Universitaet zu Kiel (Alemania), Hoegskolan i Boras (Suecia), Universitat de Barcelona (España) y Newcastle University (Reino Unido). El objetivo principal del proyecto es promover la construcción de procesos reflexivos para el DPC en alumnos a punto de acabar su formación para ser profesores de lenguas extranjeras. Para ello, los profesores en formación participan en un curso semipresencial en el que desarrollan un e-portfolio docente y realizan una investigación en el ámbito de la didáctica de lenguas extranjeras en relación con el uso y la aplicación de las tecnologías móviles u otras herramientas TIC.

A lo largo del curso, los participantes Ilevan a cabo diversas actividades centradas en la práctica reflexiva y en el uso de las TIC (herramientas de aprendizaje móvil para la enseñanza-aprendizaje de lenguas extranjeras) y el desarrollo profesional. Durante este proceso formativo, los alumnos realizan una estancia de una semana en otra universidad junto a otros profesores en formación de las demás instituciones que forman parte del proyecto. Con ello, las cinco instituciones tienen la intención de empoderar a profesores en formación a partir de una participación activa en los procesos de DPC.

Para desarrollar la experiencia derivada del proyecto, en primer lugar, presentamos los fundamentos teóricos que lo sustentan. Concretamente, nos centramos en qué se entiende por DPC y profundizamos en la necesidad de implementar acciones didácticas para incentivar la formación a lo largo de la vida, así como en las principales especificaciones de los organismos europeos que determinan la necesidad del DPC en la formación de profesores de lenguas extranjeras. A continuación, se especifican los objetivos de esta experiencia formativa. Acto seguido, se presentan la estructura y la metodología del proyecto, haciendo especial hincapié en la creación de comunidades de aprendizaje transnacional como valor innovador del mismo. Para terminar, se detalla el impacto previsto en la formación de profesores de lenguas extranjeras.

\section{El DESARROLLO PROFESIONAL CONTINUO EN LA ENSEÑANZA Y APRENDIZAJE DE LENGUAS EXTRANJERAS}

El proyecto que aquí presentamos busca promocionar la profesionalización, la innovación y la colaboración transnacional en la enseñanza-aprendizaje de lenguas extranjeras. Por profesionalización se entiende la conducta a partir de la cual los profesionales ejercen una labor como especialistas de un determinado sector (Evetts, 2009). En la formación de profesores de lenguas extranjeras, la profesionalización es comúnmente considerada como un proceso profundo y a largo plazo en el que los docentes adquieren y desarrollan críticamente conocimientos y habilidades propios de su ámbito. El propósito está en desarrollar un buen pensamiento, planificación y prácticas profesionales en cada fase de la vida 
como profesor (British Council, 2011; Day, 1999; Hayes, 2014), con un foco de atención específico en las habilidades, conocimiento, prácticas y competencias propias de su determinado perfil profesional (British Council, 2011). Asimismo, en el contexto del DPC en la formación de profesores de lenguas extranjeras, la innovación y la colaboración transnacional son aspectos centrales a partir de los cuales se debe desarrollar la formación continua (Consejo de Europa, 2009; Evetts, 2009).

La complejidad del DPC ha ido variando y sus objetivos se han ido ampliando en las últimas décadas. Según Borg (2015), el DPC se ha movido de la preocupación por las habilidades metodológicas, como los saberes prácticos relacionados con la gestión del aula o el uso de la tecnología, a una noción mucho más amplia de la práctica profesional. En este sentido, el DPC contempla en la actualidad una variedad más extensa de dimensiones, tales como la profundización en el conocimiento sobre los alumnos y el aprendizaje, el diseño de materiales, la competencia evaluativa, las cuestiones afectivas y otras habilidades como la colaboración, la construcción de redes o la reflexión. Tal y como señalan Day y Sachs $(2004,222)$, "the function of CPD may be seen to be one of three imperatives: to align teachers' practice with educational policies [...]; to improve the learning outcomes of students by improving the performance of teachers; or (less evident but more aspirational) to enhance the status and profile of the teaching profession".

La implicación de las actuales líneas de investigación en los contextos de enseñanza y los diferentes desarrollos de la política educativa juegan también un papel importante. En muchos casos, el DPC fortalece a profesores y formadores de profesores en la búsqueda de estrategias para que sean agentes de su desarrollo a partir de acciones de reflexión sobre su propia formación. Según la Comisión Europea (2013), los principales rasgos para que el desarrollo docente sea efectivo son el foco en el contenido, el aprendizaje activo, la coherencia, la duración de este y la participación colectiva. Por lo tanto, el estudio del DPC parte actualmente de una consideración pluridimensional del mismo, que abarca desde la dimensión individual, a la dimensión colectiva y colaborativa. En este sentido, Mann y Walsh (2017) hacen referencia al Desarrollo Profesional Colaborativo, subrayando su alcance cuando se trabaja "by working with other teachers who are facing similar problems, by sharing concerns and issues and by adopting an individualistic, more collectivist oriented approach to professional development" (Mann y Walsh, 2017, 107). Según Kennedy (2011), la aproximación colaborativa al DPC es un enfoque eficaz si los procesos de aprendizaje se llevan a cabo en períodos largos y en grupos reducidos de profesores. En estos procesos de DPC colectivo, es especialmente relevante el papel instrumental de la práctica reflexiva compartida.

Para profesores en formación y formadores de profesores, la integración de redes multilingües y transnacionales es crucial para fomentar la motivación, el aprendizaje intercultural, las habilidades comunicativas y, por supuesto, el desarrollo profesional (Laakkonen, 2011). Asimismo, el intercambio de ejemplos 
de buenas prácticas puede llevar a una auténtica recopilación de posibilidades efectivas de aprendizaje y enseñanza de lenguas extranjeras de las que todos los participantes se puedan beneficiar. El hecho de partir de bagajes educativos diferentes en cuestiones nacionales y de identidad implica una creación de red y, por lo tanto, de comunidades de práctica complejas y ricas para el DPC de los profesores en formación.

La importancia del DPC y la necesidad de su implementación a todos los niveles de la formación de profesores han llevado a varias instituciones a desarrollar documentos de referencia que tienen como objetivo promover los procesos de DPC entre la comunidad de docentes de lenguas extranjeras, en diferentes contextos y situaciones de formación y desarrollo docente. Entre ellos, cabe destacar en primer lugar el Portfolio europeo para futuros profesores de idiomas (PEFPI o EPOSTL, por sus siglas en inglés) (Newby et al., 2007), que está dirigido a docentes en formación inicial, así como a formadores y tutores. El objetivo de este portafolio es que su usuario reflexione sobre su propio proceso formativo en relación con las determinadas competencias docentes que va adquiriendo. La estructura del portafolio contiene una declaración personal, un conjunto de descriptores de autoevaluación centrados en diferentes áreas de contenido y un dosier de evidencias. Las áreas de contenido estriban en aspectos básicos, que van desde la reflexión sobre el contexto de enseñanza, la programación del curso y la metodología hasta competencias de enseñanza en el aula y relativas a la evaluación del aprendizaje y a la mejora del aprendizaje autónomo.

Por su parte, otras instituciones, como EAQUALS o el British Council, han elaborado documentos orientados a profesores de lenguas extranjeras en ejercicio con la intención de fomentar el DPC y establecer patrones de profesionalización. Por ejemplo, el European Profiling Grid (Mateva, Vitanova y Tashevska, 2011) se dirige tanto a profesores de lenguas extranjeras en servicio como a formadores y coordinadores académicos, ya que permite identificar al profesor con determinados niveles de dominio relativos a diversas habilidades y cualificaciones docentes. Se trata de una parrilla interactiva basada en diferentes áreas de contenido y en la que encontramos descriptores de autoevaluación para que el docente se pueda ubicar en un determinado estadio. Este documento pretende facilitar en principio la delineación de planes de desarrollo y de necesidades de formación. De esta forma, la parrilla se flexibiliza antes distintas finalidades del DPC (evaluativas y certificativas, entre otras) y los roles de los actores responsables de dichos procesos (docentes, formadores y coordinadores académicos).

Por otro lado, el CPD Framework (British Council, 2015) es un documento que se orienta a profesores en ejercicio, aunque no en exclusiva de lenguas extranjeras. De manera similar a la European Profiling Grid, este documento permite al docente situarse en un nivel de desarrollo específico, de entre cuatro que se establecen, con el fin de trazar un plan concreto de actuación. El contenido de este marco se centra en diferentes áreas relativas a prácticas profesionales, a las cuales corresponden distintas descripciones de ejemplos de buena práctica y actividades. 


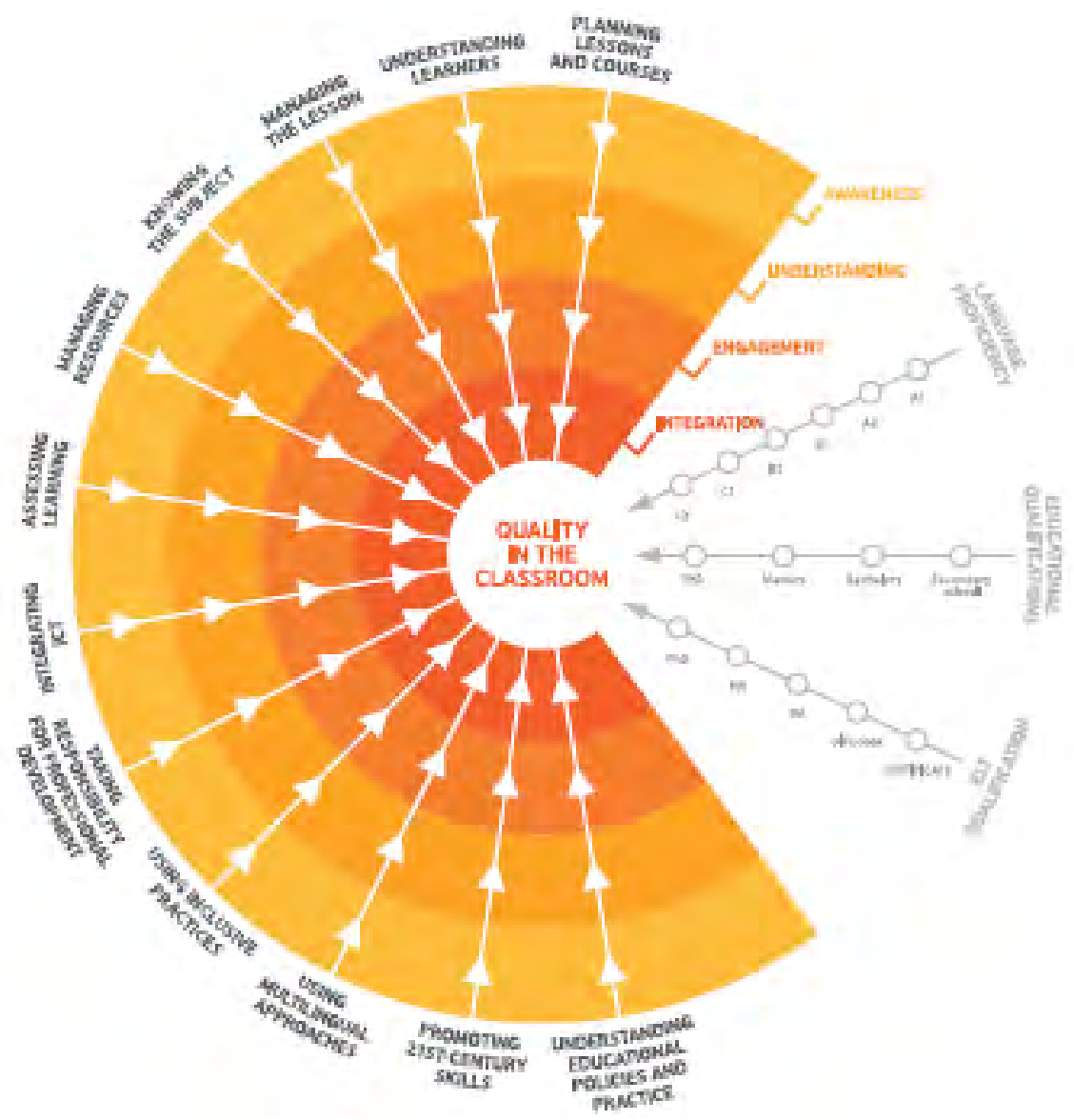

Figura 1. Síntesis de prácticas profesionales del CPD framework (British Council, 2015)

Por último, destacamos un documento que guarda relación con las competencias digitales de los docentes, el European Framework for Digital Competences of Educators, o DIGCOMPEDU (Redecker, 2017). Esta herramienta proporciona una descripción sistemática de las competencias digitales, que abarcan tanto las competencias pedagógicas y profesionales del docente -en formación inicial o en ejercicio- como las competencias del aprendiente. Incluye dimensiones básicas, organizadas en categorías, como el proceso de enseñanza-aprendizaje, la evaluación, la gestión de los recursos y las estrategias para facilitar el aprendizaje centrado en el alumno. De las competencias profesionales del docente, se destaca la atención especial a aspectos como la colaboración profesional, la práctica reflexiva y el DPC digital. Los descriptores de las distintas competencias digitales se distribuyen en un modelo progresivo de niveles: A1 (Awareness), A2 (Explorer), B1 (Integration), B2 (Expertise), C1 (Leadership), C2 (Innovation). 


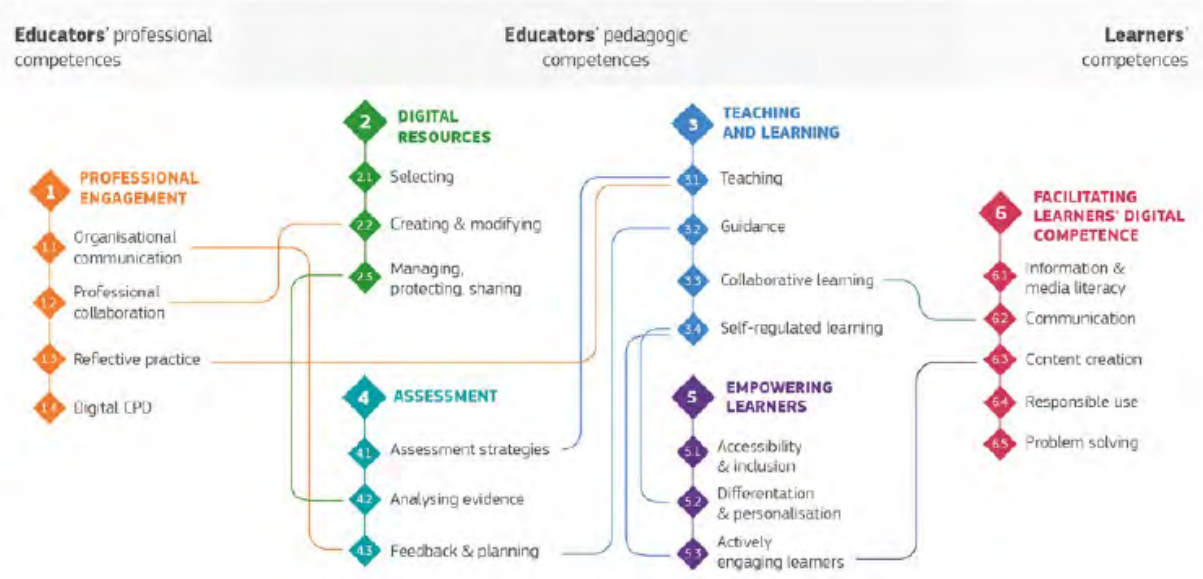

Figura 2. Síntesis de competencias digitales de Digcompedu (Redecker, 2017)

Los documentos institucionales presentados dan cuenta de la importancia del DPC en la formación de profesores de lenguas extranjeras y de la necesidad de sistematización de niveles o estadios de aprendizaje. La reflexión derivada del desarrollo competencial en la sociedad del siglo XXI tiene implicaciones fundamentales para el DPC. Diversos estudios a gran escala han demostrado que el profesor tiene una gran importancia en los procesos y resultados de aprendizaje de los alumnos (Hattie, 2009, 2012; OCDE, 2014) algo que ya había sido afirmado anteriormente (Consejo de Europa, 2009; Lortie, 1977; Slavin, 1994). El DPC es, por lo tanto, una realidad comprendida como un proceso esencial a través del cual todo profesor construye su aprendizaje a lo largo de la vida y aprende a adaptarse o a transformarse con los rápidos cambios de la sociedad actual (OCDE, 2014; Taylor, 2011).

La importancia del DPC se ha incrementado a lo largo de los últimos 30 años, periodo de tiempo en el cual el desarrollo profesional ha estado presente en el ámbito de la investigación educativa. Sin embargo, se ha constatado la ausencia de perspectivas teóricas e investigaciones de carácter empírico sobre el DPC en contextos académicos específicos en los que se lleva a cabo un desarrollo profesional, como, por ejemplo, en el ámbito de la enseñanza-aprendizaje de lenguas (Allwright, 2001; Andrews, 2007; Borko, 2004; Crandall y Christison, 2016; Freeman y Johnson, 1998), a menudo limitada a su propia tradición (Richards y Farrell, 2005). Además, la integración y la combinación de modos creativos de enseñanza y aprendizaje a través del uso de tecnologías o el establecimiento de redes profesionales no han sido todavía parte del DPC de profesores y formadores de profesores, los cuales aún están centrados mayoritariamente en la búsqueda de aproximaciones pedagógicas efectivas en relación con esta nueva cultura de enseñanza y aprendizaje (Burden y Kearney, 2016; Estapa, Pinnow y Chval, 2016; McLoughlin y Lee, 2009; UNESCO, 2012). Un ejemplo de estas aproximaciones a nuevas pedagogías efectivas es el aprendizaje móvil o m-learning (Burden, Kearney, Schuck y Aubusson, 2015), entendido como los 
procesos de enseñanza a través del uso de tecnologías móviles y transportables como teléfonos inteligentes o tabletas, que constituyen una parte integral de la profesión docente. El tratamiento del m-learning para el desarrollo del DPC de los profesores en formación es uno aspecto de necesario cumplimiento. Esta sociedad, en las que las herramientas TIC y el aprendizaje móvil están tan presentes, requiere de profesores que tengan en cuenta las nuevas pedagogías efectivas y las adopten en sus procesos de desarrollo profesional.

Nuestro proyecto indaga en la necesidad de establecer parámetros para el DPC fundamentados en el desarrollo de comunidades de práctica colaborativas transnacionales y en el tratamiento del aprendizaje móvil. El proyecto, pues, está pensado para que los futuros profesores de lenguas extranjeras puedan acceder a una formación basada en el intercambio de ideas y experiencias, más allá, de las propias fronteras y en un contexto europeo de cara a la constante transformación profesional que exige la sociedad del siglo XXI.

\section{Objetivos del proyecto}

El objetivo del proyecto PROPIC Europa es promover el DPC del profesorado de lenguas extranjeras a partir de la elaboración de un programa de estudio interdisciplinar, orientado a la investigación y fundamentado en el uso creativo de las tecnologías móviles. En consecuencia, se vincula el DPC de los profesores en formación al intercambio de experiencias formativas de diversa índole a partir del establecimiento de redes sociales transnacionales que puedan consolidarse como comunidades de práctica.

\section{DeSCRIPCIÓN DEL PROYECTO}

\section{Estructura y metodología}

El proyecto tiene una duración de tres años, (septiembre de 2017 - junio de 2020), en los cuales se realizan tres ediciones del curso, una por cada año del proyecto, en cada una de las cinco instituciones participantes (véase figura 3). En cada curso, participan un máximo de 12 alumnos, por lo que se espera que, tras las tres ediciones, un total de 180 profesores en formación participen en la formación implicada en PROPIC Europa. Junto a los 14 profesores formadores, docentes de las cinco universidades participantes, hacen un total de 194 participantes en el proyecto.

El programa de estudio se realiza mediante una serie de acciones de aprendizaje en las que se aúnan práctica reflexiva, conocimiento y uso de la tecnología móvil y desarrollo profesional a través de la investigación. Los aspectos que se tratan en este programa son lo suficientemente amplios para que se puedan trazar especificaciones formativas variadas. La heterogeneidad de los contextos implicados en el proyecto, tantos como instituciones involucradas, supone la necesidad de dar versatilidad al programa de estudio. Así, mientras que en algunos centros, como en la Universidad de Barcelona, el curso se integra dentro del programa 
de Máster en Formación de Profesores de Español como Lengua Extranjera, en otros, como en las Universidades de Kiel y de Karlsruhe, es un curso optativo que implica la obtención de créditos para los estudios de los grados de enseñanza de lenguas extranjeras. Dadas las características contextuales mencionadas, cada universidad desarrolló su propio curso de formación adaptado a sus alumnos, es decir, para alumnos que se están formando para ser profesores de lenguas extranjeras en diferentes contextos de enseñanza: educación primaria, secundaria o para adultos.

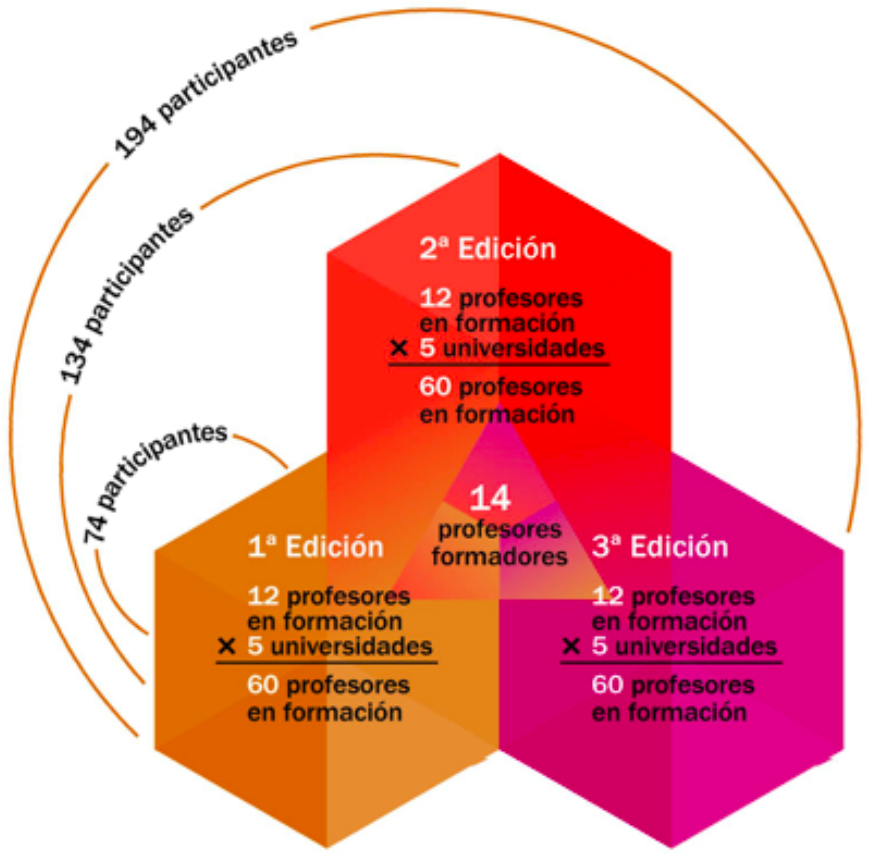

Figura 3. Esquema general de las ediciones del proyecto y número de participantes

El curso se desarrolla en tres partes. En una primera parte, que se realiza en la universidad de origen, el foco de atención está en la práctica reflexiva, la investigación y el uso de las tecnologías móviles para el DPC. En la segunda parte del curso, correspondiente a una semana presencial que tiene lugar en una universidad diferente a la de origen, los alumnos participan en actividades colaborativas en las que se estimula el desarrollo de la práctica reflexiva, la implementación de las tecnologías móviles para la enseñanza-aprendizaje de lenguas extranjeras o el desarrollo de la competencia docente a través de la observación de clases. Esta segunda parte es clave para comprender la transnacionalidad del proyecto: los 12 profesores en formación de cada universidad se distribuyen entre las cuatro universidades restantes, las universidades de destino: tres profesores en formación por universidad. En consecuencia, cada institución recibe tres estudiantes del resto de universidades (véase figura 4a). 

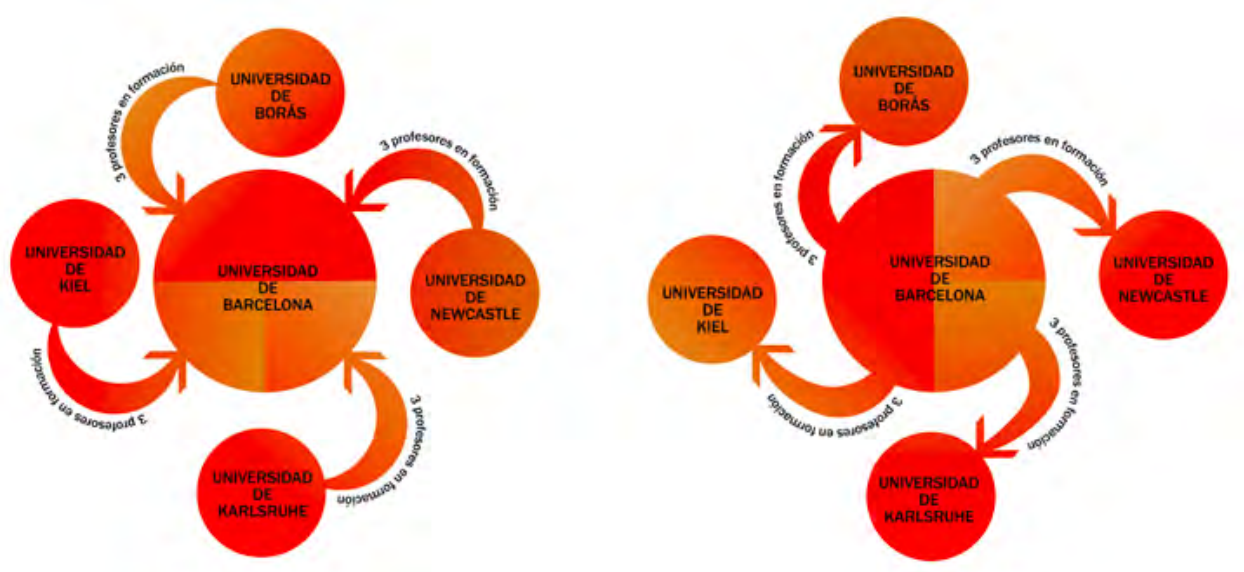

Figura 4a. Flujo de participantes durante la segunda fase de las universidades de origen a la universidad de destino

Del mismo modo, cada universidad envía a tres estudiantes a cada una de las otras universidades (véase figura $4 b$ ).
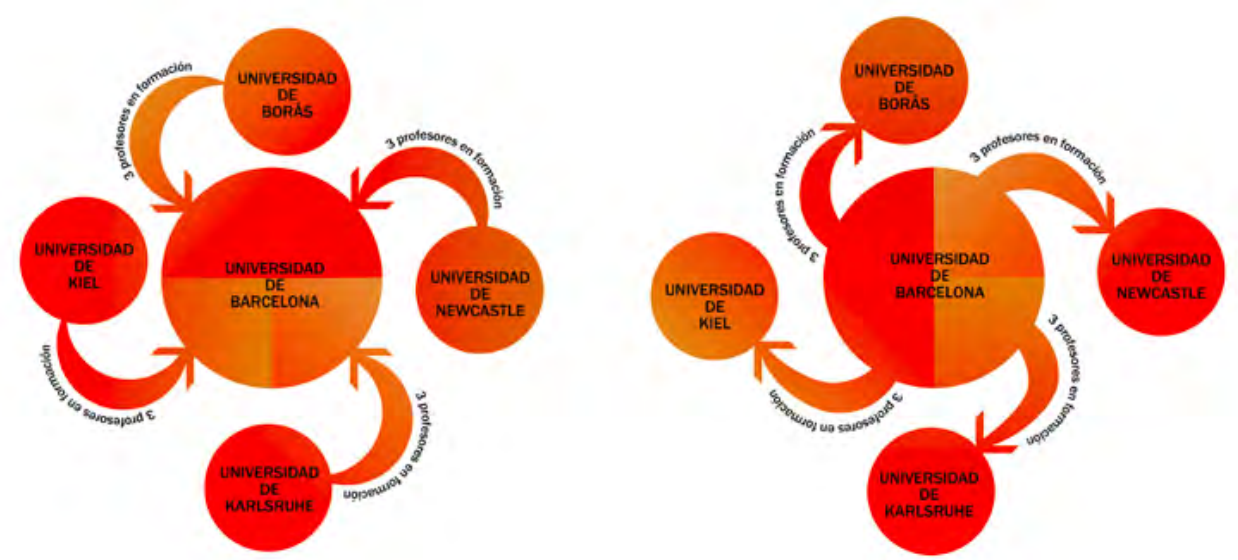

Figura 4b. Flujo de participantes durante la segunda fase de la universidad de origen a las universidades de destino

Por último, en la tercera parte del curso, que se desarrolla en las universidades de origen, los estudiantes comparten las experiencias de aprendizaje durante la segunda parte del curso y presentan sus productos finales: un portafolio digital docente y un proyecto personal de investigación en el aula de lenguas extranjeras o bien de diseño de propuestas didácticas basadas en el uso de las tecnologías móviles. 
Los contenidos del curso, correspondientes a los objetivos de la formación, se desarrollan a través de un conjunto de 8 ebooks con los que se busca que los docentes en formación adquieran herramientas básicas para el DPC y el desarrollo de la práctica reflexiva. A través de la lectura interactiva de los ebooks, los profesores aprenden a crear y desarrollar un portafolio digital, a realizar observaciones de clases, a elaborar vídeos educativos y libros electrónicos y a utilizar herramientas de aprendizaje móvil para diseñar actividades de aprendizaje. Algunos ebooks son de lectura obligatoria, mientras que otros son opcionales o recomendados según la universidad. Sus contenidos son trabajados en diferentes talleres y seminarios a lo largo de las diferentes fases del curso.

La metodología del curso está fundamentada en un trabajo tanto presencial en el aula como autónomo fuera de ella. Las sesiones presenciales varían en función de la universidad que imparte el curso, mientras que el trabajo autónomo se fundamenta en la lectura de los diferentes libros electrónicos y la realización de las actividades de aprendizaje propuestas en estos materiales. Algunas de estas actividades se realizan de forma colaborativa tanto entre miembros de la misma universidad como entre miembros de universidades diferentes. Par este cometido, se utiliza la red social Slack, plataforma online que se utiliza también para la comunicación entre todos los participantes del proyecto.

\section{La creación de comunidades de aprendizaje transnacional como innovación didáctica}

En la Europa del siglo XXI, la necesidad de abrir fronteras pasa por compartir y contrastar experiencias que vayan más allá de nuestras propias realidades nacionales. Es por ello que la transnacionalidad del proyecto y su potencialidad para sustentar la creación de comunidades de aprendizaje es el aspecto más innovador de esta formación. En un plano personal, el proyecto ofrece la posibilidad de entrar en contacto con otros estudiantes en otros países en situaciones formativas similares. En un plano profesional, el proyecto permite crear una comunidad de aprendizaje transnacional a partir de la cual desarrollar el propio DPC.

La configuración de la comunidad de aprendizaje vinculada al programa de estudio se inicia a través de la red social Slack (véase figura 5). La plataforma permite a sus participantes desarrollar hilos conversacionales con fines pedagógicos, sociales y administrativos. Los estudiantes crean y participan en estos hilos para, entre otras acciones, presentarse y establecer un primer contacto con compañeros de otras universidades, realizar actividades reflexivas conectadas con los contenidos de los ebooks o presentar las ideas de sus proyectos finales. La herramienta Slack permite, pues, comenzar a establecer la comunidad de aprendizaje antes de que los alumnos lleguen a las universidades de destino en las que realizarán su estancia. La faceta presencial de la comunidad es central en esta semana de experiencias con otros docentes de similares características, inquietudes y perfil profesional. El intercambio producido es enriquecedor desde el punto de vista formativo: a partir del contraste y la reflexión sobre las propias experiencias formativas, se establece la posibilidad de generar conocimiento que implique un DPC más fructífero, además de llevar a cabo prácticas interculturales a partir de las cuales reflexionar sobre las diversas realidades docentes de los diferentes países. 


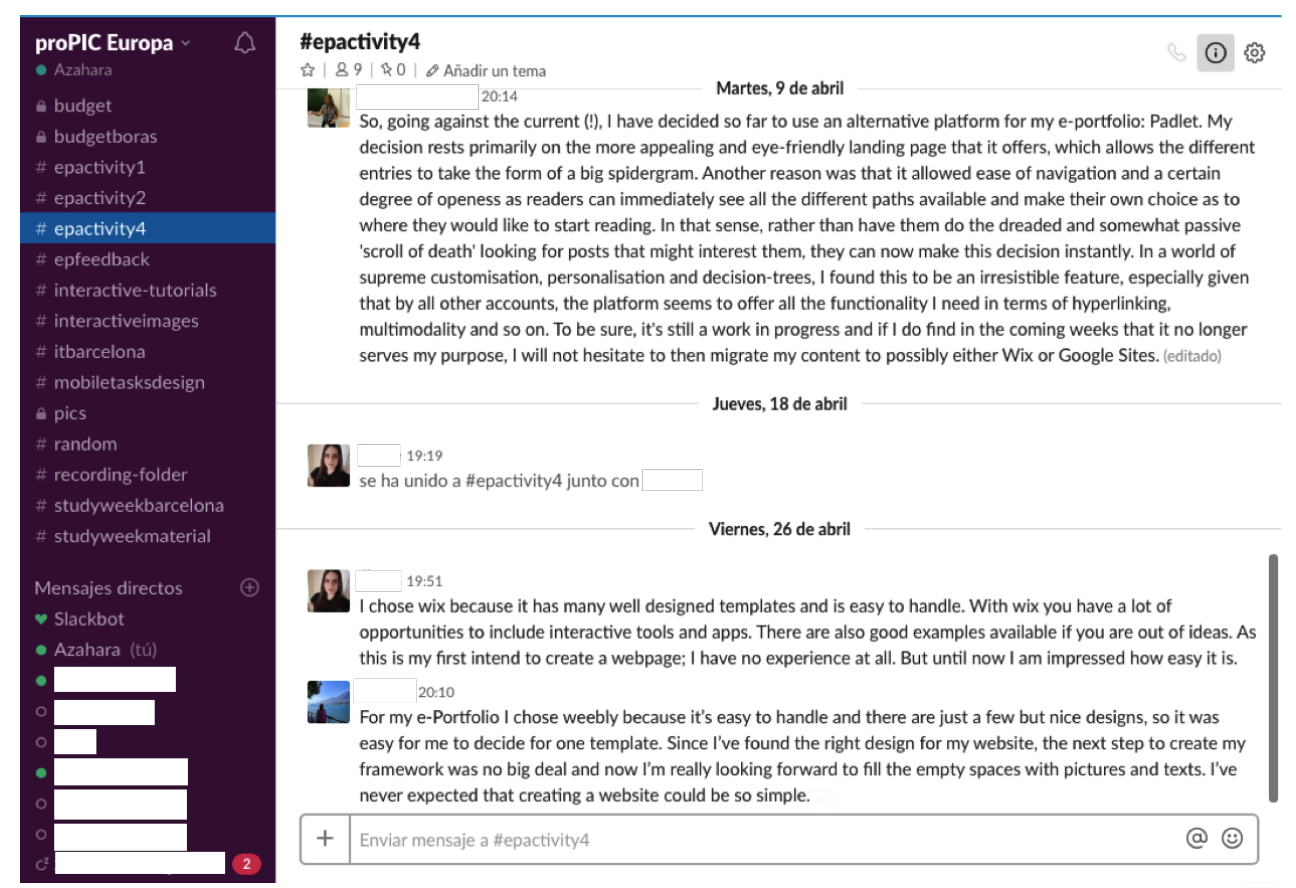

Figura 5. Ejemplo de actividad reflexiva a través de Slack entre participantes de diferentes universidades

Desarrollo profesional docente a través del uso de las TIC

La presencia de las herramientas TIC en este proyecto no solo es relevante en la creación y desarrollo de la comunidad transnacional de aprendizaje a distancia, sino también en su uso por parte de los profesores en formación a lo largo del curso con la intención de desarrollar la competencia digital docente. En esta línea, en algunos de los talleres presenciales, los alumnos pudieron, por ejemplo, observar cómo se pueden utilizar los lectores de códigos QR para crear actividades interactivas y aprendieron a crear imágenes multimodales con la aplicación Genially. En las actividades en línea y a distancia, se utilizó, entre otras herramientas, Flipgrid, una aplicación que permite grabar y compartir vídeos para que los compañeros puedan comentarlos en cualquier momento y lugar a través de sus teléfonos móviles.

Por otro lado, la práctica reflexiva también fue desarrollada a partir del uso de herramientas digitales. Un ejemplo de ello fue el uso de la aplicación Video Enhanced Observation (VEO). Esta aplicación permite grabar con una tableta y, a partir de un sistema de etiquetaje, determinar aspectos de la clase grabada. Tanto el vídeo grabado como las marcas establecidas a partir del sistema de etiquetaje y notas derivadas que se puedan escribir, quedan grabadas en la aplicación y pueden ser utilizadas para, posteriormente, comentar qué ha ocurrido en la clase. Para conocer cómo funcionaba la aplicación, los alumnos del curso pudieron grabar una sesión de clase y 
etiquetar el vídeo, focalizando la atención en algún aspecto de la práctica docente que les llamara la atención. Posteriormente, se llevaron a cabo varias actividades a partir de las cuales los alumnos pudieron reflexionar sobre la sesión de clase observada, además de sobre la viabilidad de la herramienta de observación de clases.

\section{Resultados Y CONCLUSIONES}

La consecución de los objetivos fijados en el proyecto ya está generando un impacto en los diferentes agentes implicados: los futuros profesores de lenguas extranjeras, los profesores implicados de las diferentes universidades y por extensión en la formación de profesores de lenguas extranjeras que se lleva a cabo en estos centros. A más largo plazo, este impacto también recaerá en los alumnos a los que estos profesores en formación tendrán la oportunidad de enseñar.

En los profesores en formación, este impacto se centra en cuatro ejes: desarrollar su propia agencia como profesionales en el campo de la educación lingüística, adquirir nuevas habilidades con y mediante la tecnología móvil y el aprendizaje transnacional entre iguales, fomentar las colaboraciones transnacionales y las relaciones con compañeros docentes en el extranjero y, por último, desarrollar una actitud global de profesionalismo e innovación. Así, este proyecto ha de capacitar a los profesores para hacer un uso creativo de la tecnología móvil, así como propiciar una implementación de la misma en su futura labor como docentes de lenguas extranjeras. Además, se pretende despertar la curiosidad y la inquietud hacia formas innovadoras de enseñanza y de aprendizaje, de forma que los docentes se conviertan en diseminadores de buenas prácticas en el uso de tecnología móvil en el aula de lenguas extranjeras y de enfoques de enseñanza innovadores.

Por su parte, puede afirmarse que los 14 profesores formadores que participan en el proyecto también han desarrollado su competencia docente al aprender de sus pares y de la experiencia del contexto directo con alumnos de universidades diferentes, lo que ha generado una comunidad de práctica con la intención de atender a las necesidades de los alumnos en un contexto de enseñanza global. En relación con las organizaciones participantes, se sigue trabajando para que estas puedan:

- Desarrollar y mejorar el programa de estudios de cada institución participante hacia los objetivos generales del proyecto, es decir, profesionalismo, innovación y colaboración transnacional;

- Crear y fortalecer vínculos existentes entre instituciones y empoderar a las instituciones participantes para llegar a otros socios internacionales y partes interesadas.

- Divulgar los resultados del proyecto en sus universidades y en los foros especializados de sus respectivos países.

Creemos en la importancia del impacto del proyecto a largo plazo, el que se conseguirá una vez estos 180 profesores en formación ya estén desarrollando su labor docente. El proyecto tiene el potencial de tener una incidencia duradera en las escuelas donde los futuros maestros Ileguen a trabajar. El objetivo general, la im- 
plementación de la DPC como un aspecto fundamental y decisivo de la formación docente, no se detendrá con la graduación de los estudiantes, sino que se prolongará en el tiempo por el trabajo continuo en los e-portafolios. Asimismo, la recopilación de estudios de caso y productos realizados por los estudiantes y el repositorio de libros electrónicos ya está proporcionando recursos para redefinir las formaciones en las que estamos colaborando.

Tras la finalización de la primera edición del proyecto, se llevó a cabo una primera aproximación a las percepciones de los profesores en formación sobre sus experiencias como participantes en el proyecto formativo. La recogida de datos se efectuó mediante un cuestionario en línea de preguntas cerradas, abiertas y de valoración de escala Lickert, en la cual participaron 28 estudiantes involucrados en la primera edición del curso (mayo de 2018). Los participantes en el cuestionario tienen edades comprendidas entre los 20 y los 50 años, proceden de las diferentes instituciones involucradas en el proyecto y presentan un perfil académico diverso, que comprende la enseñanza de lenguas extranjeras (inglés, español y sueco) en educación primaria, secundaria y para adultos.

Los resultados preliminares del cuestionario subrayan como elementos más valorados de la estructura del proyecto la estancia en otra universidad y la utilidad de las herramientas digitales presentadas en el proyecto, valoradas como muy útiles por el $88 \%$ de los encuestados. Respecto a la estancia en otra universidad, los aspectos mejor valorados por los estudiantes son la efectividad y la claridad del input presentado en estas estancias, la oportunidad para utilizar diferentes tecnologías y la posibilidad de realizar visitas a escuelas. El apoyo tanto pedagógico como técnico recibido para desarrollar la colaboración internacional en estas estancias ha sido especialmente subrayado por los participantes: el $100 \%$ de los encuestados destaca la calidad de la interacción promovida entre los profesores en formación de la institución de destino durante la segunda parte del curso. Los participantes destacan que el trabajo conjunto con otros profesores en formación de otros países ha enriquecido el desarrollo de ideas y estrategias para implementar herramientas TIC como docentes y ha promovido la reflexión hacia un uso crítico de tales herramientas en el aula.

Tras indagar en los datos obtenidos al inicio del proyecto, ya podemos vislumbrar resultados positivos, particularmente en los profesores en formación participantes. Las sesiones de trabajo conjunto les han permitido compartir experiencias, inquietudes y retos, además de permitirles poder observar otros modos de trabajar en la formación del profesorado y la enseñanza de lenguas extranjeras. Por su parte, gracias a la estancia en una universidad extranjera con compañeros de origen diverso, los profesores en formación han tomado conciencia de la importancia de establecer una red propia de formación que propicie el intercambio de ideas, inquietudes y necesidades formativas a través de la reflexión conjunta e individual. Esa red o comunidad de aprendizaje ha podido evolucionar hacia una comunidad de práctica transnacional. Por otro lado, 14 profesores formadores participantes en el proyecto han consensuado procedimientos didácticos, contenidos y actividades que han servido como base para la creación de un curso de formación de profesores, cuyo resultado ha culminado en la creación de los ocho ebooks mencionados con anterioridad. 
Tomando como punto de partida estos resultados, observamos que es posible fomentar el DPC de futuros profesores de lenguas extranjeras a partir de proyectos formativos basados en la interdisciplinariedad, la transnacionalidad y el enfoque reflexivo, investigador y creativo hacia el uso de las tecnologías móviles. No obstante, por el estadio en el que se encuentra el proyecto, es necesario más tiempo para, a medio plazo, poder verificar el impacto del mismo en ediciones posteriores del proyecto, poniendo en relación el análisis de las percepciones de los participantes con un análisis más exhaustivo de los productos llevados a cabo por los estudiantes y su desempeño en las actividades formativas. Además, a largo plazo, cabría plantearse profundizar en el impacto de esta formación sobre el aprendizaje de los futuros alumnos de los profesores en formación participantes.

Las diferentes instituciones participantes mantienen objetivos y retos comunes en cuanto a la formación del profesorado de lenguas extranjeras. Compartirlos, analizar cómo abordarlos y trabajar sobre ellos es lo que permite una formación de la identidad docente que pueda evolucionar a partir de una base sólida común y de forma paralela entre los diferentes participantes. Es por todo ello que creemos en la necesidad de proyectos de este tipo, que acompañan al alumnado en su formación inicial y en su desarrollo profesional posterior.

\section{REFERENCIAS BIBLIOGRÁFICAS}

Allwright, D. (2001). Three major processes of teacher development and the appropriate design criteria for developing and using them. En B. Johnson y S. Irujo (Eds.), Research and Practice: Voices from the field (pp. 115-133). Minneapolis: Center for Advanced Research on Language Acquisition.

Andrews, S. (2007). Language Teacher Awareness. Cambridge: CUP.

Borg, S. (2015).Teaching for Success. Contemporary perspectives on continuing professional development. Londres: British Council.

Borko, H. (2004). Professional Development and Teacher Learning: Mapping the Terrain. Educational Researcher, 3(8), 3-15. Recuperado de https://pdfs.semanticscholar.org/f317/c2912db1c09387a5eb17dfa66b063956afba.pdf.

British Council (2011). Going forward: Continuing Professional Development for English Language Teachers in the UK. Londres: British Council. Recuperado de https://englishagenda.britishcouncil.org/sites/default/files/filefield_paths/b413_cpd_for_teachers_v2.pdf.

British Council (2015). Continuing Professional Development (CPD) Framework for Teachers. Londres: British Council. Recuperado de https://www.teachingenglish. org.uk/sites/teacheng/files/CPD\%20framework\%20for\%20teachers_WEB.PDF.

Burden, K. y Kearney, M. (2016). Future Scenarios for Mobile Science Learning. Research in Science Education, 46(2), 287-308. https://doi.org/10.1007/s11165-016-9514-1.

Burden, K., Kearney, M., Schuck, S. y Aubusson, P. (2015). Viewing Mobile Learning from a Pedagogical Perspective.ALT-J Association for Learning Technology Journal, 20(3), 1-17. http://doi.org/10.3402/rlt.v20i0.14406. 
Comisión Europea (2013). Supporting teacher competence development for better learning outcomes. European Commission-Education \& Training. Recuperado de http://ec.europa.eu/assets/eac/education/404_en.htm.

Consejo de Europa (2009). Council conclusions of 26 November 2009 on the profesional development of teachers and school leaders. Oficial Journal of the European Union, 12.12.2009 C 302/6 Recuperado de https://eur-lex.europa.eu/LexUri Serv/ LexUriServ.do?uri=OJ:C:2009:302:0006:0009:EN:PDF.

Crandall, J. y Christison, M. (Eds.) (2016). Teacher Education and Professional Development in TESOL: Global Perspectives. Londres: Routledge.

Day, C. y Sachs, J. (2004). International Handbook on the Continuing Professional Development of Teachers. Maidenhead: Open University Press.

Estapa, A., Pinnow, R. y Chval, K. (2016). Video as a professional development tool to support novice teachers as they learn to teach English language learners. The New Educator, 12(1), 85-104. http://doi.org/10.1080/1547688X.2015.1113350.

Evetts, J. (2009). The management of professionalism: A contemporary paradox. En S. Gewirtz, P. Mahony, I. Hextall y A. Cribb (Eds.), Changing Teacher Professionalism: International Trends, Challenges and Ways Forward (pp. 19-30). London: Routledge.

Freeman, D. y Johnson, K. (1998). Reconceptualizing the knowledge-base of language teacher education. TESOL Quarterly, 32(3), 397-417.

Hattie, J. (2009). Visible learning: A synthesis of over 800 meta-analyses relating to achievement. Londres: Routledge.

Hattie, J. (2012). Visible learning for teachers. Londres: Routledge.

Hayes, D. (Ed.) (2014). Innovations in the continuing professional development of English language teachers. Londres: British Council.

Kennedy, A. (2011). Collaborative continuing professional development (CPD) for teachers in Scotland: aspirations, opportunities and barriers. European Journal of Teacher Education, 34(1), 25-41. https://doi.org/10.1080/02619768.2010.53 4980.

Laakkonen, I. (2011). Personal learning environments in higher education language courses: an informal and learner-centered approach. En S. Thouësny y L. Bradley (Eds.), Second language teaching and learning with technology: views of emergent researchers (pp. 9-26), Dublin: Research-publishing.net. http://doi.org/10.14705/ rpnet.2011.00004.

Lortie, D. (1977). Schoolteacher: A Sociological Study. Chicago: University of Chicago Press.

Mateva, G., Vitanova, A. y Tashevska, S. (2011). The European profiling grid: A user guide. Recuperado de http://www.epg-project.eu/wp-content/uploads/The-EPGPDF-publication_EN.pdf. 
McLoughlin, C. y Lee, M. (2009). Personalised Learning Spaces and Self-Regulated Learning: Global examples of Effective Pedagogy. Proceedings ascilite Auckland 2009 (pp. 639-645). Auckland, Nueva Zelanda: University of Auckland. Recuperado de https://pdfs.semanticscholar.org/5e22/eb07f0ad773c38e55dde11b296dbb6983364.pdf.

Newby, D., Allan, R., Fenner, A-B., Jones, B., Komorowska, H. y Soghikyan, K. (2007). EPOSTL - A reflection tool for language teacher education. Strasbourg: Council of Europe Publishing. Recuperado de http://epostl2.ecml.at/LinkClick. aspx?fileticket=Odz4pL2JvAk=\&t.

OCDE (2014). TALIS 2013 Results: An International Perspective on Teaching and Learning. Paris: OECD Publishing. http://doi.org/10.1787/9789264196261-en.

Richards, J. y Farrell, T. (2005). Professional Development for Language Teachers. Strategies for Teacher Learning. Cambridge: CUP.

Redecker, C. (2017). European Framework for the Digital Competence of Educators: DigCompEdu. En Y. Punie (Ed.), EUR 28775EN. Luxembourg: Publications Office of the European Union. http://doi.org/10.2760/159770.

Slavin, R. (1994). Using Student Team Learning. Baltimore, MD: Johns Hopkins University, Center for Social Organization of Schools.

Taylor, S. (2011). Behavior Basics: Quick Behavior Analysis and Implementation of Interventions for Classroom Teachers. The Clearing House, 84(5), 197-203. http:// doi.org/10.1080/00098655.2011.568988.

UNESCO (2012). Education for sustainable development: sourcebook. UNESCO education sector. Learning \& Training Tools, 4. Recuperado de https://unesdoc. unesco.org/ark:/48223/pf0000216383. 